\title{
Replica of Sink Ability Test with Beeker Test
}

\author{
Sreenivasa Rao Pagolu* \\ Assistant General Manager, Production, Maintenance and Utilities, BMR Industries Pvt. Ltd., Nellore, India \\ *Corresponding author: srpagolu@gmail.com
}

\begin{abstract}
The sink-ability test is to be carried out frequently for the pellets made during winter season, since surface tension of water during low temperature is high. Pellets have to break surface tension to sink into the water. This test will be carried out morning hours at around 6:00 Am at ponds. During every startup of production or during new formulation, this test is mandatory. Normally ponds are away from factory, carrying out of this test is became a big issue to the quality control team to confirm the sink ability. This is to be made easy by making replica of pond test.
\end{abstract}

Keywords: Ability, Beeker, Pond, Replica, Sink.

\section{Introduction}

The Standard practice of sink ability test is to draw the samples from production line, then move to pond to test. If the pellets pass the test, then the production may be continued, otherwise root cause to find out for failure, then corrective and preventive actions are to be carried out.

\section{Sample Collection}

Samples are drawn from production line at pre-defined intervals for various batches as well as from various lines and sealed enclosed cover or in a container to protect from environmental effects/atmosphere conditions. The same counter samples are to be drawn to test for moisture verification as well as for future cross verification of test.

The test samples are to be taken to the pond during early morning hours where the temperatures are low as well as the surface tension of water is high. The pellets are to be taken by hand and through into the water in air flow direction and record sinking time. If the pellets are sinking with in one or two minutes' maximum, will be treated pass the test, otherwise based on time taken to sink will be considered for sink-ability. The same will be informed to production, either the production is carried out or need a correction. If correction is made either in process or in formulation or in any other manner, the test to be repeated. During repeat test the atmospheric temperature increases or surface tension of water decreases, then the conducting the test may not give right results.

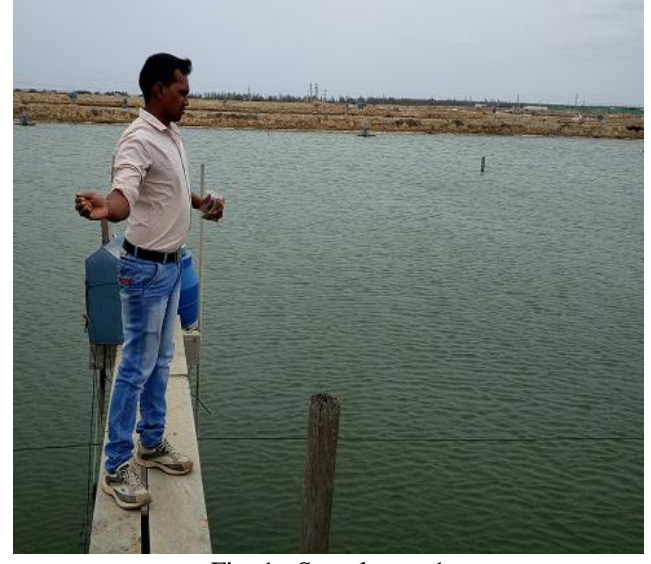

Fig. 1. Sample test 1

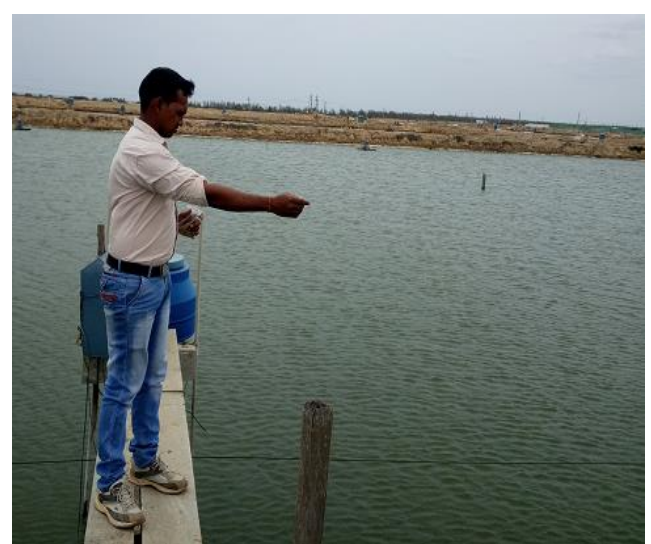

Fig. 2. Sample test 2

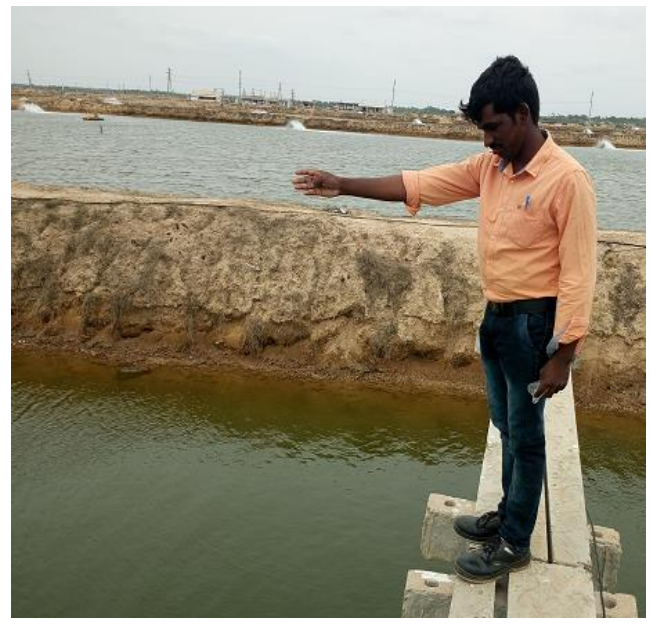

Fug. 3. Sample test 3 


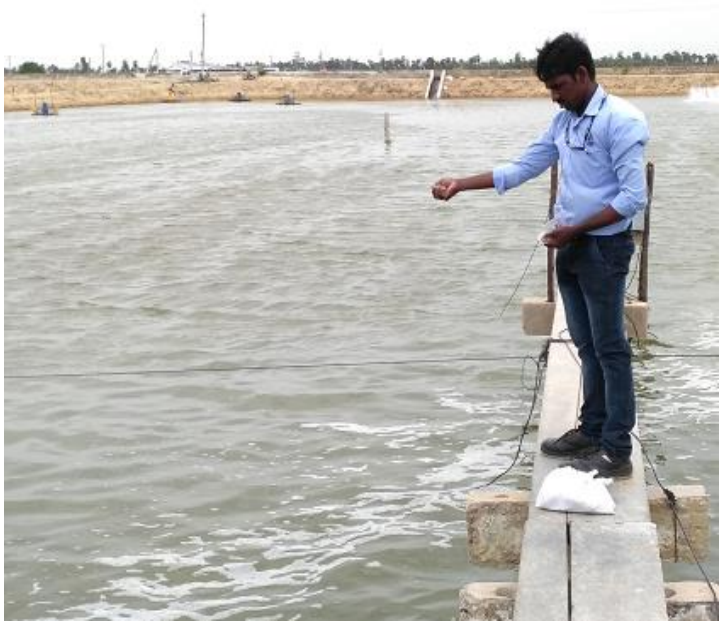

Fig. 4. Sample test 4

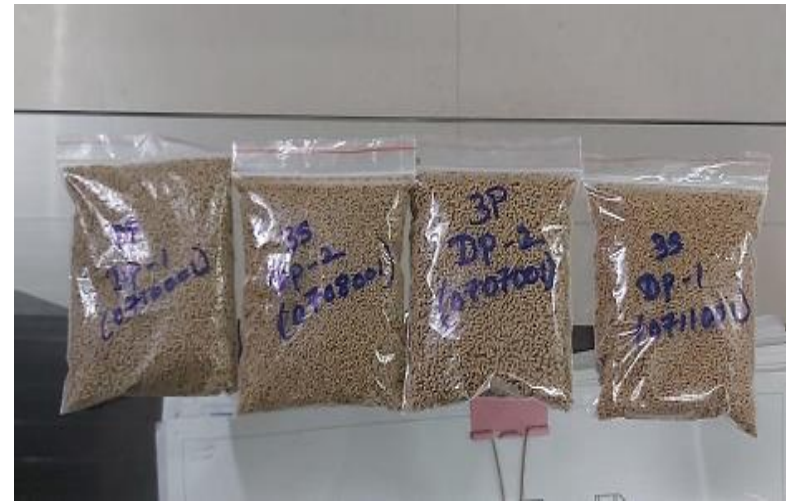

Fig. 5. Counter sample 1

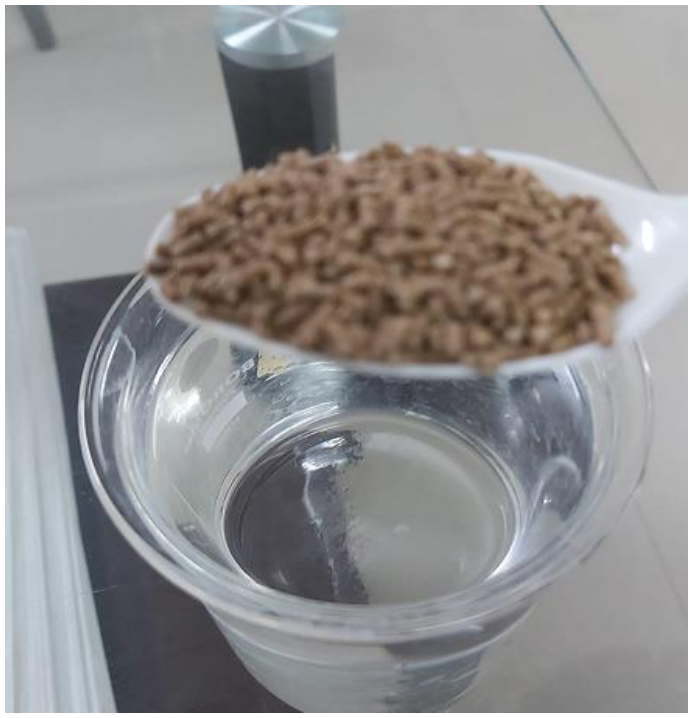

(a)

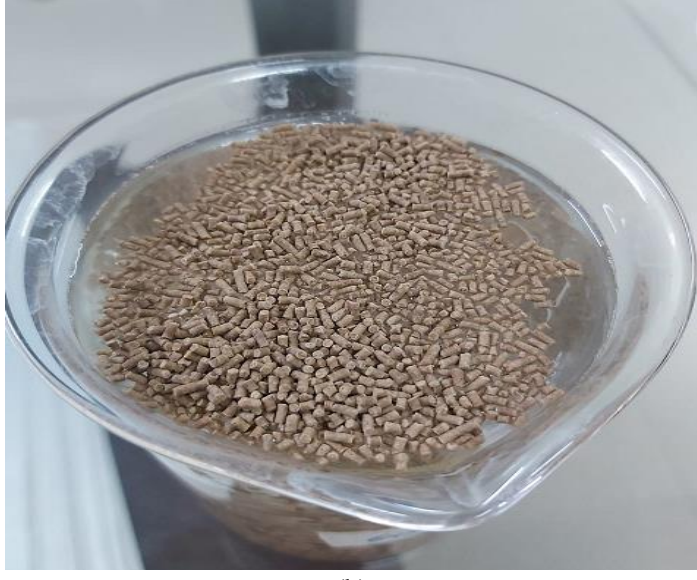

(b)

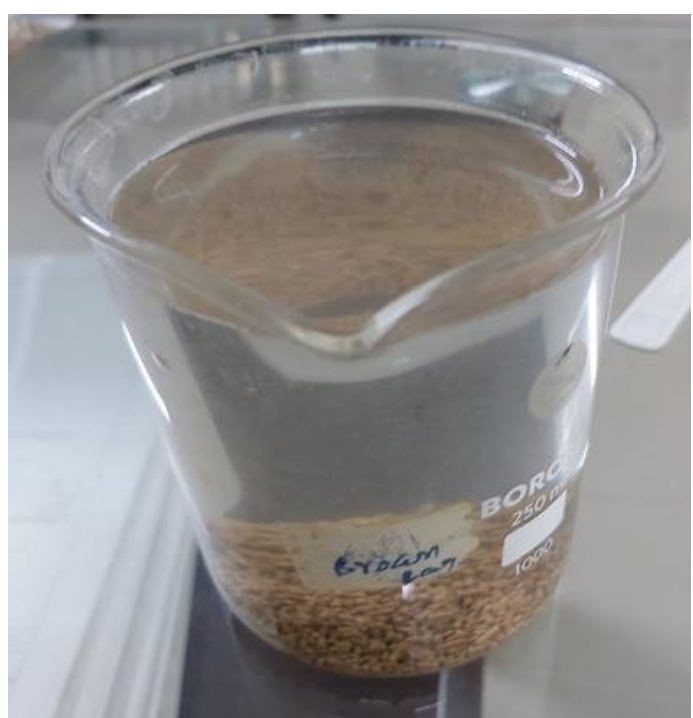

(c)

Fig. 6. Beeker test 1

\section{Test Procedure}

The counter samples are taken for Beeker test from the same batch where the samples are drawn for pond test 1,2,3,4. The Beeker is filled with water with lower temperature like 16 degrees centigrade. The table spoon used to pour the pellets in the Beeker slowly like a layer on water shown in the figure 6(b). Wait until the complete feed is sinking. Record the Time. Repeat the tests for sample 2, 3 and 4.

Table 1

Comparison between pond test and Beeker test

\begin{tabular}{|l|c|c|}
\hline Samples & Pond test time in sec & Beeker test time in sec \\
\hline Sample no 1 & 80 & 40 \\
\hline Sample no 2 & 60 & 30 \\
\hline Sample no 3 & 50 & 27 \\
\hline Sample no 4 & 60 & 29 \\
\hline
\end{tabular}

\section{Analysis}

Compare the data between pond test and Beeker test. The corelation between pond test and Beeker test is Time taken for 
sinking of pellets. For the sample 1 , pond test time is $1 \mathrm{~min} 20$ sec whereas Beeker test time is $40 \mathrm{sec}$., for the sample 2, pond test time is $1 \mathrm{~min}$ and Beeker test time are $30 \mathrm{sec}$. For the sample 3 , pond test time is $50 \mathrm{sec}$ and Beeker test time are $27 \mathrm{sec}$., For the sample 4, pond test time is 1 min whereas Beeker test time is $29 \mathrm{sec}$. It indicates that the time taken in the pond test and Beeker test are correlated. The time taken for Beeker test is $1 / 2$ of pond test time.

\section{Conclusion}

By above data analysis, we may consider that the pond test may be replaced with Beeker test for day-to-day analysis, whereas pond may be done at pre-defined intervals like weekly once to compare with Beeker test. If any major deviations are found, then that the Beeker test is to be revalidated by using lower temperature water.

\section{References}

[1] A. I. Vogel, "Practical Organic Chemistry," Third edition (Longman, London), 1974.

[2] L. B. Reddy, "Test Procedures manual," BMR industries Library edition, 2018. 